\title{
BMJ Open Multiple system atrophy in Hokkaido, Japan: a prospective registry study of natural history and symptom assessment scales followed for 5 years
}

\author{
Masaaki Matsushima (D , ${ }^{1}$ Ichiro Yabe, ${ }^{1}$ Ken Sakushima, ${ }^{1}$ Yasuhiro Kanatani, ${ }^{2,3}$ \\ Naoki Nishimoto, ${ }^{4}$ Takeshi Matsuoka, ${ }^{5}$ Jun Sawada, ${ }^{6}$ Haruo Uesugi, ${ }^{7,8}$ \\ Kazuya Sako, ${ }^{9}$ Asako Takei, ${ }^{10}$ Akiko Tamakoshi, ${ }^{11}$ Shun Shimohama, ${ }^{12}$ \\ Norihiro Sato, ${ }^{13}$ Seiji Kikuchi, ${ }^{14}$ Hidenao Sasaki ${ }^{1,15}$
}

To cite: Matsushima M, Yabe I, Sakushima K, et al. Multiple system atrophy in Hokkaido, Japan: a prospective registry study of natural history and symptom assessment scales followed for 5 years. BMJ Open 2021;11:e045100. doi:10.1136/ bmjopen-2020-045100

- Prepublication history for this paper is available online. To view these files, please visit the journal online (http://dx.doi. org/10.1136/bmjopen-2020045100).

Received 29 September 2020 Revised 12 December 2020 Accepted 18 December 2020

Check for updates

(C) Author(s) (or their employer(s)) 2021. Re-use permitted under CC BY-NC. No commercial re-use. See rights and permissions. Published by BMJ.

For numbered affiliations see end of article.

Correspondence to Dr Masaaki Matsushima; mmasaaki@huhp.hokudai.ac.jp

\section{ABSTRACT}

Objectives Multiple system atrophy (MSA) is a refractory neurodegenerative disease, but novel treatments are anticipated. An accurate natural history of MSA is important for clinical trials, but is insufficient. This regional registry was launched to complement clinical information on MSA.

Setting Patient recruitment started in November 2014 and is ongoing at the time of submission. The number of participating facilities was 66 . Postal surveys were sent to medical facilities and patients with MSA in Hokkaido, Japan.

Participants After obtaining written consent from 196 participants, 184 overview surveys and 115 detailed surveys were conducted.

Primary and secondary outcome measures An overview survey evaluated conformity to diagnostic criteria and a detailed survey implemented an annual assessment based on the Unified Multiple System Atrophy Rating Scale (UMSARS).

Results At the time of registration, $58.2 \%$ of patients were diagnosed with cerebellar symptoms predominant type MSA (MSA-C) and $29.9 \%$ were diagnosed with parkinsonism predominant type MSA (MSA-P). UMSARS Part IV score of 4 or 5 accounted for $53.8 \%$ of participants. The higher the UMSARS Part IV score, the higher the proportion of MSA-P. At baseline, levodopa was used by 69 patients (37.5\%) and the average levodopa dose was $406.7 \mathrm{mg} /$ day. The frequency of levodopa use increased over time. Eleven cases changed from MSA-C to MSA-P during the study, but the opposite was not observed. Information about survival and causes of death was collected on 54 cases. Half of deaths were respiratory-related. Sudden death was recorded even in the group with UMSARS Part IV score of 1. Conclusions This study is the first large-scale prospective MSA cohort study in Asia. MSA-C was dominant, but the use of antiparkinsonian drugs increased over the study period. Changes from MSA-C to MSA-P occurred, but not vice versa.

\section{INTRODUCTION}

Multiple system atrophy (MSA) is an adult-onset, refractory, progressive
Strengths and limitations of this study

- This study is the first large-scale prospective multiple system atrophy (MSA) registry system in Asia.

- Our research provides useful clinical information on the differences between cerebellar symptoms predominant type MSA and parkinsonism predominant type MSA.

- Conformity to diagnostic criteria was confirmed, and symptom assessment scales such as the Unified Multiple System Atrophy Rating Scale and the Multiple System Atrophy Health-Related Quality of Life scale were continuously evaluated by postal survey once a year.

- Use of drugs to treat symptoms was investigated over time.

- One of the limitations of this study was that MSA was diagnosed clinically and not pathologically confirmed.

neurodegenerative disease characterised by cerebellar ataxia, parkinsonism and autonomic dysfunction. ${ }^{2}$ The causes of MSA remain unknown, and radically improved therapies have not been developed. Patients with MSA usually require canes or wheelchairs within 5 years and die within 10 years of disease onset. Clues to the pathogenesis of MSA, such as $\alpha$-synuclein aggregation in oligodendroglia, ${ }^{3}$ tubulin polymerisation-promoting protein impairment, ${ }^{4}$ inflammation, ${ }^{5}$ mitochondrial dysfunction and the V393A variant of $C O Q 2{ }^{6}{ }^{6}$ have been revealed in recent years. Clinical trials of growth hormone, ${ }^{7}$ riluzole,${ }^{8}$ rifampicin, ${ }^{9}$ minocycline,${ }^{10}$ rasagiline ${ }^{11}$ and epigallocatechin gallate $^{12}$ have been conducted. Unfortunately, significant benefits were not obtained. However, the development of novel disease-modifying therapies, such as mesenchymal stem cell therapy, ${ }^{13}$ 
induced pluripotent stem cell therapy ${ }^{14}$ and coenzyme Q10 therapy, ${ }^{15}$ is expected. Appropriate sample sizes needed to produce statistically significant results must be ensured before these agents can be used in clinical trials on patients with MSA.

In Europe and the USA, several prospective cohort studies have been conducted, ${ }^{816-18}$ but few long-term and large-scale studies. Moreover, the primary symptoms of MSA differ between Europe/USA and Japan; in the former, parkinsonism predominates, whereas in the latter cerebellar symptoms predominate. Retrospective studies have been conducted in Japan and Asia, ${ }^{19-21}$ but prospective epidemiological data remain insufficient. Therefore, in Japan and Asia, the collection of natural history data on disease progression and investigations into natural history modifiers are essential for evaluation of treatment interventions and preparation of MSA guidelines.

To solve this problem, we first conducted a crosssectional study in Hokkaido. ${ }^{22}$ Some points of background are relevant: (1) Hokkaido is a single autonomous island with low population movement; (2) there was a sufficient number of patients with MSA in Hokkaido for an epidemiological study, namely 647 , by national data collected in 2014; and (3) the many neurologists in Hokkaido ensured a high intrinsic rate of cooperation from the Hokkaido Prefecture. In this prior study, parkinsonian or autonomic-dysfunction-at-onset MSA had a poor prognosis and elderly-onset MSA had shorter survival times.

Next, we thought it necessary to set up a system for long-term tracking of a large prospective cohort. We thus organised a regional MSA registry (the Hokkaido Rare Disease Consortium for MSA or HoRC-MSA) for foundation construction of clinical trials going forward. The purpose of the present study was to clarify the natural history of MSA and to provide basic data for clinical trials.

\section{MATERIALS AND METHODS}

\section{Participants and evaluation}

Patient recruitment started in November 2014. At study start, we used the national registry data in Hokkaido to locate patients, asking about their intention to participate through their respective medical institutions (not only by certified neurologists, but by all medical institutions in Hokkaido) and taking written consents. Since 2016, we have been accepting registration requests directly from the patients and families. The electronic data capture system started operation in 2018. New registrations are presently being accepted on an ongoing basis. Postal surveys were also conducted, in which doctors and patients responded to a questionnaire. The overview survey concerned the type of MSA, impact on activities of daily living (ADL; by the Unified Multiple System Atrophy Rating Scale (UMSARS ${ }^{23}$ ) Part IV), medication and items related to the diagnostic criteria (second consensus statement) for MSA. ${ }^{24}$ Participants were asked to respond to a detailed survey if their disease severity was $1-4$ on UMSARS Part IV. The detailed survey gathered scores on UMSARS Part II-Motor, the Multiple System Atrophy Health-Related Quality of Life (MSA-QoL) scale,${ }^{25}$ height, body weight, and symptom assessment scale items that changed quickly, which were named ICLEMSA (Items That Change Largely in Early-Stage Multiple System Atrophy; ie, handwriting, finger taps, transfers, standing with feet together, turning the trunk, turning $360^{\circ}$, gait and body sway; total score $=36) .{ }^{26}$ Regarding the type of MSA, in addition to parkinsonism predominant type (MSA-P) versus cerebellar symptoms predominant type (MSA-C), which was determined by the attending physician, other items of the overview survey and UMSARS Part II were comprehensively evaluated.

The survey was conducted once a year. In this paper, we analyse the results up to the fifth evaluation, based on the reply data up to October 2019. The doctor or a family member contacted the research office when the patient changed institutes or died. Information on survival and causes of death was collected from the patient's family and attending physician and analysed.

\section{Statistical analysis}

Data were managed as connectable and anonymised. Student's t-test was performed on continuous variables and Fisher's exact test was performed on discrete variables. The significance level was $<0.005$, and two-sided test was performed. Statistical analysis was conducted using JMP Pro V.14.0.0 (SAS Institute, Cary, North Carolina, USA). Score changes were evaluated using the standardised response mean (SRM). SRM was obtained by dividing the average score change by the SD of score change. An SRM >0.8 was considered large, 0.5-0.8 moderate and $<0.5$ small. ${ }^{27}$ We interpreted a larger SRM as indicative of more rapid symptom progression. KaplanMeier curves were constructed to summarise information on patient deaths.

\section{Cohort description}

\section{Patient and public involvement}

In this study, we recruited Japanese patients with MSA through neurologists and medical institutions in all regions of Hokkaido, Japan. Participants exchanged documents directly with the research office by mail and returned the written consents and completed questionnaires. The Department of Health and Welfare, Hokkaido Government and Sapporo City Public Health Office cooperated in public relations for patients with MSA. The results were disseminated to study participants by newsletters from the research office.

\section{RESULTS}

Between November 2014 and October 2019, 254 patients with MSA requested the informed consent form of the registry. Of these, written consent was obtained from 196, 184 (female=97) overview surveys were conducted and 115 detailed surveys were performed (figure 1A). The number of participating facilities was 66 . In the first year, 
A

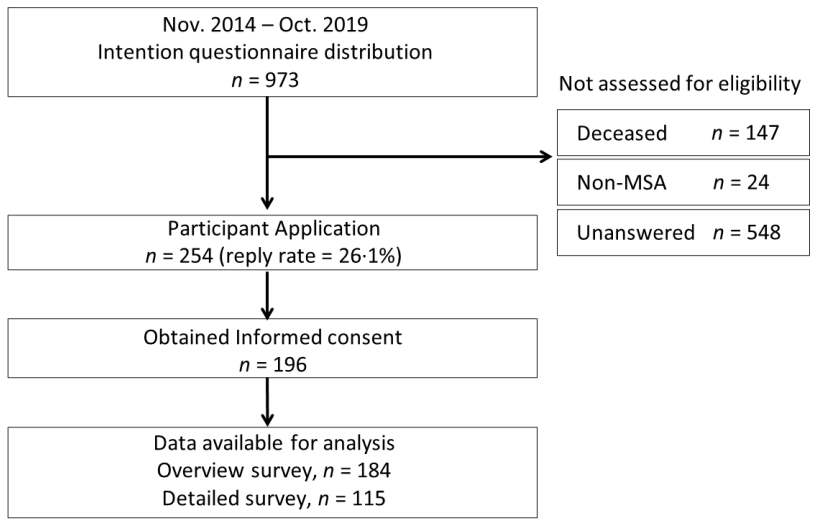

B

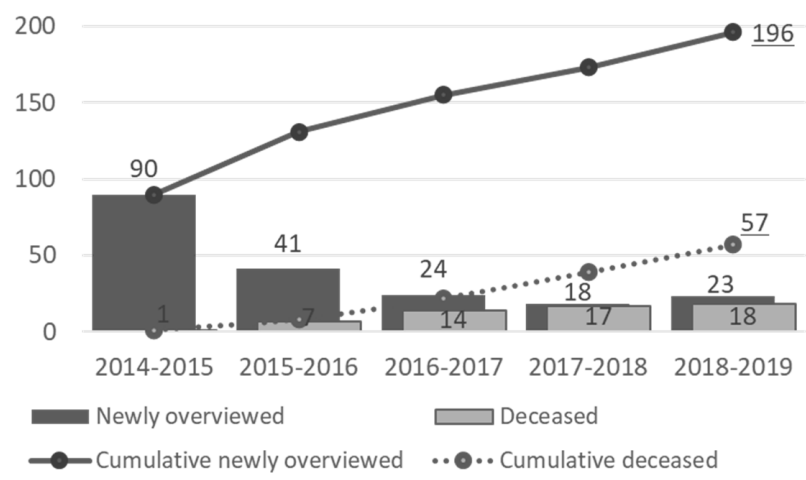

Figure 1 Flow diagram of the study. (A) Overview of registration. (B) New overview surveys and deaths each year from the start of the study. Solid line: cumulative new overviewed participants; dotted line: cumulative deaths. MSA, multiple system atrophy.

90 overview data were registered and a certain number of new registrations occurred each year. However, the number of cases that dropped out due to death gradually increased (figure 1B). During the above period, 104 patients performed the second-year evaluation, 71 performed the third-year evaluation, 41 performed the fourth-year evaluation and 17 performed the fifth-year evaluation. Patients' background at the time of registration is shown in table 1, with $58.2 \%$ (107) MSA-C and $30.0 \%$ (55) MSA-P. 'Other' (meaning that cerebellar ataxia and parkinsonian symptoms were comparable or difficult to measure) was at $12.0 \%$ (22). The MSA-C:P ratio was from $54.8 \%$ to $27.9 \%(57 / 29)$ in the second-year evaluation, from $47.9 \%$ to $33.8 \%(34 / 24)$ in the thirdyear evaluation, from $51.2 \%$ to $29.3 \%(21 / 12)$ in the fourth-year evaluation, and from $58.8 \%$ to $23.5 \%$ (10/4) in the fifth-year evaluation. Of the patients $75.5 \%$ showed some parkinsonism at the initial evaluation (table 1). The disease type percentage stratified by UMSARS Part IV score at the time of the initial evaluation is shown in figure 2. We noted a tendency for lower proportions of MSA-C to associate with poorer ADL.

In some cases, the dominant symptoms changed during the study period. The type of MSA changed from MSA-C to MSA-P in 11 cases, the mean transition time being $23.7 \pm 13.0$ (median 17 ) months after registration and 58.5 \pm 41.2 (median 49) months after diagnosis. At this time, UMSARS Part IV scores deteriorated from 3.7 (average of previous assessment before change) to 4.6 (average of evaluation at the time of change). As a comprehensive judgement, we observed no obvious change in the reverse direction, from MSA-P to MSA-C.

In the present data, 17 patients were able to confirm the change from possible MSA to probable MSA, with an average of $2.4 \pm 0.7$ years after registration (median 2 years) and an average of $4.6 \pm 3.0$ years after diagnosis (median 3 years). Overall, the proportion of probable MSA at the first evaluation was $76 \%$, but all 17 cases that could be followed up to the fifth evaluation were patients with probable MSA. On the other hand, we recorded nine patients who remained possible MSA; initially all of them were MSA-C, of whom two patients changed to MSA-P later and two died. The reason for their remaining possible MSA cases was that, despite the presence of ataxia, their autonomic dysfunction did not meet the diagnostic criteria.

The UMSARS Part IV scores ADL in outline. Thus, 1 (independent) was initially $6.0 \%, 2$ was $21.4 \%, 3$ was $18.7 \%$, 4 was $31.3 \%$, and 5 (bedridden) was $22.5 \%$. This distribution worsened over time. By the fifth evaluation, $94.1 \%$ of patients had UMSARS Part IV score of 4 or 5. UMSARS Part II, MSA-QoL motor scores and ICLEMSA scores also increased (figure 3A). Since the number of cases that could be completed up to the fifth evaluation was small, figure 3 was drawn from the data of 16 cases that could be followed continuously up to the fourth evaluation. Looking at the progress of MSA-C and MSA-P, MSA-P had higher scores than MSA-C in almost all evaluations of UMSARS Part II (figure 3B). In MSA-QoL, MSA-P and MSA-C scored almost the same, and in ICLEMSA, MSA-C had slightly higher scores (figure 3C,D). The proportion of MSA-P increased initially, but changed little over the course of 5 years. As shown in figure 3E, the SRM values were high in UMSARS, ICLEMSA and MSA-QoL in the first to second evaluations. The SRM of ICLEMSA remained almost the same at the second to third, the third to fourth and the fourth to fifth evaluations. SRM of UMSARS and MSA-QoL fluctuated. Body mass index (BMI) did not change significantly.

At baseline, the thyrotropin-releasing hormone analogue taltirelin was used in 117 patients $(64.3 \%)$ and levodopa in 75 patients $(40.8 \%)$. The average dose of levodopa was $406.7 \mathrm{mg} /$ day. In MSA-P cases, levodopa was used in $81.5 \%$. The frequency of levodopa use in all cases increased to $41.3 \%$ at the second evaluation, $47.9 \%$ at the third evaluation, $61.0 \%$ at the fourth evaluation and $70.6 \%$ at the fifth evaluation. The frequency of taltirelin use changed less: about $60 \%$ in all cases and about $25 \%$ in MSA-P cases $(27.6 \%, \mathrm{n}=8$, second evaluation; $20.8 \%$, $\mathrm{n}=5$, third evaluation; $25.0 \%, \mathrm{n}=3$, fourth evaluation; and $50.0 \%, \mathrm{n}=2$, fifth evaluation). Other therapeutic agents for Parkinson's disease were used in 38 cases, selegiline in 12 cases, pramipexole in 10 cases and amantadine in 8 cases (table 1 ). As shown in figure $3 \mathrm{~F}$, the average 
Table 1 Characteristics of study patients at registration and overview survey

\begin{tabular}{|c|c|c|c|c|c|}
\hline & Total & MSA-P & MSA-C & Others & $P$ value \\
\hline Patients, $\mathrm{n}$ & 184 & 55 & 107 & 22 & \\
\hline Gender, female (\%) & $97(53)$ & $34(62)$ & $51(48)$ & $12(55)$ & 0.192 \\
\hline Months from diagnosis to registration, mean $\pm S D$ & $49.7 \pm 47.7$ & $41.8 \pm 39.6$ & $45.4 \pm 44.6$ & $98.9 \pm 62.4$ & $<0.001$ \\
\hline Probable MSA, n (\%) & $140(76)$ & $48(87)$ & $74(69)$ & $18(82)$ & 0.028 \\
\hline Cerebellar oculomotor disturbance, $\mathrm{n}(\%)$ & $106(58)$ & $21(38)$ & $77(72)$ & $8(36)$ & $<0.001$ \\
\hline Limb ataxia, $\mathrm{n}(\%)$ & $152(83)$ & $36(65)$ & $104(97)$ & $12(55)$ & $<0.001$ \\
\hline Ataxic gait, n (\%) & $137(74)$ & $28(51)$ & $99(93)$ & $10(45)$ & $<0.001$ \\
\hline Any parkinsonism, n (\%) & $139(76)$ & $55(100)$ & $65(61)$ & $19(86)$ & $<0.001$ \\
\hline Retropulsion, n (\%) & $95(52)$ & $48(87)$ & $48(45)$ & $13(59)$ & $<0.001$ \\
\hline Any autonomic symptoms, n (\%) & $161(88)$ & $48(87)$ & $93(87)$ & $20(91)$ & 0.953 \\
\hline Urine catheter placement, $\mathrm{n}(\%)$ & $44(24)$ & $11(20)$ & $18(17)$ & $15(68)$ & $<0.001$ \\
\hline Frequent urination, $\mathrm{n}(\%)$ & $77(42)$ & $25(45)$ & $49(46)$ & $3(14)$ & $<0.001$ \\
\hline Urinary incontinence, $\mathrm{n}(\%)$ & $71(39)$ & $26(47)$ & $41(38)$ & $4(18)$ & $<0.001$ \\
\hline Erectile dysfunction, $\mathrm{n}(\%)$ (only in male: 87) & $39(21)$ & $11(20)$ & $26(24)$ & $2(9)$ & 0.185 \\
\hline Orthostatic hypotension, n (\%) & $37(20)$ & $17(31)$ & $19(18)$ & $1(5)$ & 0.057 \\
\hline Systolic blood pressure decrease, mean \pm SD & $18.8 \pm 22.0$ & $23.4 \pm 21.6$ & $16.8 \pm 22.5$ & $17.0 \pm 12.4$ & 0.339 \\
\hline Diastolic blood pressure decrease, mean \pm SD & $6.7 \pm 15.8$ & $10.3 \pm 14.7$ & $5.9 \pm 16.4$ & $-5.6 \pm 4.6$ & 0.085 \\
\hline Levodopa, n (\%) & $75(41)$ & $47(85)$ & $18(17)$ & $10(45)$ & $<0.001$ \\
\hline Selegiline, n (\%) & $12(7)$ & $11(20)$ & $1(1)$ & $0(0)$ & $<0.001$ \\
\hline Pramipexole, n (\%) & $10(5)$ & $6(11)$ & $1(1)$ & $3(14)$ & 0.002 \\
\hline Amantadine, n (\%) & $8(4)$ & $7(13)$ & $0(0)$ & $1(5)$ & $<0.001$ \\
\hline Other drugs, $n(\%)$ & $21(11)$ & $13(24)$ & $6(6)$ & $2(9)$ & 0.004 \\
\hline For orthostatic hypotension, n (\%) & $46(25)$ & $14(25)$ & $27(25)$ & $5(23)$ & 1 \\
\hline Droxidopa, n (\%) & $33(18)$ & $13(24)$ & $15(14)$ & $5(23)$ & 0.474 \\
\hline Midodrine, n (\%) & $26(14)$ & $6(11)$ & $17(9)$ & $3(14)$ & 0.679 \\
\hline Other drugs, n (\%) & $23(13)$ & $2(4)$ & $20(19)$ & $1(5)$ & 0.084 \\
\hline For dysuria, n (\%) & $67(36)$ & $17(31)$ & $44(41)$ & $6(27)$ & 0.111 \\
\hline Silodosin, n (\%) & $11(6)$ & $2(4)$ & $8(7)$ & $1(5)$ & 0.738 \\
\hline Solifenacin, n (\%) & $8(4)$ & $2(4)$ & $6(6)$ & $0(0)$ & 0.669 \\
\hline Distigmine, n (\%) & $8(4)$ & $1(2)$ & $6(6)$ & $1(5)$ & 0.573 \\
\hline Other drugs, n (\%) & $41(22)$ & $12(22)$ & $25(23)$ & $4(18)$ & 0.435 \\
\hline
\end{tabular}

'Others' is defined as patients difficult to diagnose as either MSA-C or MSA-P due to similar levels of parkinsonism and ataxia or due to being bedridden.

Student's t-test was performed on continuous variables and Fisher's exact test was performed on discrete variables.

MSA, multiple system atrophy; MSA-C, cerebellar symptoms predominant type MSA; MSA-P, parkinsonism predominant type MSA; UMSARS, Unified Multiple System Atrophy Rating Scale. 


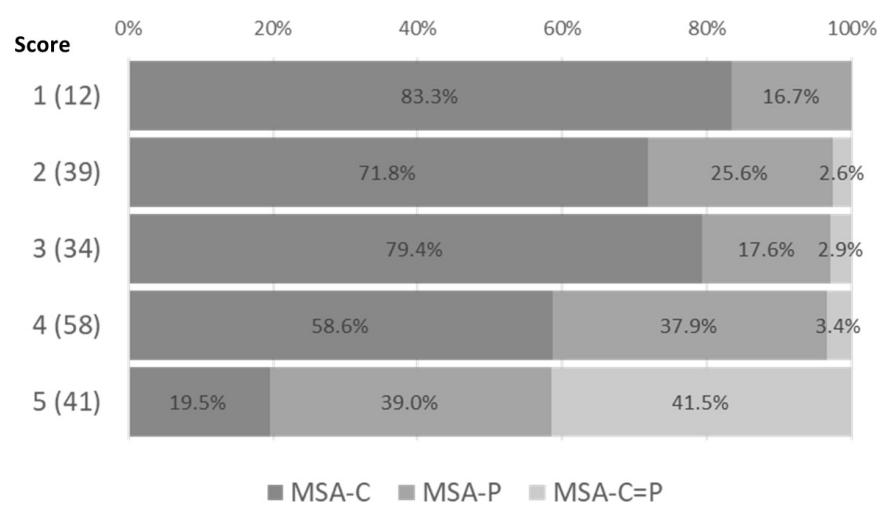

Figure 2 Disease type percentages stratified by UMSARS Part IV score at the time of registration. The number in parentheses refers to the number of patients. MSA, multiple system atrophy; MSA-C, cerebellar symptoms predominant type MSA; MSA-C=P, cerebellar ataxia and parkinsonian symptoms equally; MSA-P, parkinsonism predominant type MSA; UMSARS, Unified Multiple System Atrophy Rating Scale.

UMSARS Part II score tended to be high in the group using levodopa. Vasopressors were used in 45 patients $(24.7 \%)$ as a symptomatic treatment for autonomic dysfunction. In particular, droxidopa was used in 33 cases and midodrine in 26 cases. Medication for dysuria was prescribed to 59 patients $(32.4 \%)$. Among them, silodosin was used in 11 cases, solifenacin in 8 cases and distigmine in 8 cases (table 1). Multiple vasopressors were used in 20 cases $(11.0 \%)$.

In the study period, 63 deaths were confirmed. Of those, 56 patients were able to confirm the data of the overview survey. Information on the cause of death was collected in 54 cases. More than half of the deaths were respiratory-related, such as respiratory dysfunction, apnoea and pneumonia (figure 4A). Autopsy was performed in only one case. Although it was difficult to count sudden deaths accurately, eight patients with MSA were reported to have died unexpectedly. On the KaplanMeier curves, over about 4 years, the group with UMSARS Part IV score of 1-3 appeared to have a relatively better prognosis than that with UMSARS Part IV score of 4-5 (figure 4B). Analysis was also performed separately for findings of MSA-C or MSA-P at the time of registration; MSA-P had poorer prognosis, but the difference did not reach significance (figure 4C). Since few cases survived 50 months after enrolment, we based the Kaplan-Meier analyses on the data up to 48 months. Eight of the deaths had changed their disease type post registration, all changing from MSA-C to MSA-P.

\section{DISCUSSION}

This study (HoRC-MSA) is the first large-scale prospective cohort MSA study in Asia and its significance is great. No prospective cohort containing as many MSA-C cases has been reported. Our research may provide useful clinical information on the differences between MSA-C and MSA-P, and is relevant to a population of more than four billion Asians. HoRC-MSA has been successful due to the cooperation of many patients, their families, doctors, healthcare professionals and governments in Hokkaido. There was registration from existing patients with MSA in the first year, and from the second year onwards a certain number of patients with newly diagnosed MSA were registered sequentially (figure 1B). Although the symptoms of MSA often worsen in a short period of time, leading to transfer to a care facility, the exchange of questionnaires via patients featured in this study may be able to suppress dropouts caused by the transferring physician.

No prospective cohort study of MSA has reported on so many cases over a long period. The largest previously reported study tracked 175 cases for 5 years. ${ }^{16-18}$ The patient background reported here was similar to that of retrospective studies published in Japan and Asia. ${ }^{19-22}$ MSA-C is relatively more prevalent in Japan and Asia. What was initially expected was that the proportion of MSA-P would increase with time. ${ }^{19}$ In fact, MSA-P did not clearly increase, but the frequency of levodopa use did increase over time and the effect of parkinsonism was expected to increase. In this prospective study, a number of patients transitioned from MSA-C to MSA-P, with a median of 49 months after diagnosis, similar to what has been reported by previous retrospective studies. ${ }^{19} 28$ This change in the predominant symptoms largely coincided with the deterioration of ADL to the stage of wheelchair use. ${ }^{20}$ On the other hand, no patients clearly changed from MSA-P to MSA-C. This might indicate the possibility striatonigral degeneration in MSA pathogenesis could delay after cerebellar degeneration, but we could not confirm the pathological support in this study. This is a challenge for future studies. Although striatonigral degeneration was reported to have a poor prognosis, ${ }^{22}$ MSA-P cases showed poorer survival than MSA-C cases in this study. The background to this was that MSA-P was already registered at an advanced state (higher frequencies of probable diagnostic category $(87 \%$ vs $69 \%)$ and orthostatic hypotension ( $31 \%$ vs $18 \%$ ) in MSA-P than MSA-C). The group that here changed from MSA-C to MSA-P tended to have a shorter survival time than the remaining MSA-C cases. These points have not been mentioned in previous reports. In addition to the new findings presented in this paper, we can expect further MSA epidemiological data to be forthcoming due to the continuing nature of this registry study.

Patients enrolled in this registry were relatively advanced cases. It can be said that the initial patient recruitment based on the national registry system was a drawback (more than half of patients scored over 4 at the time of the initial evaluation). In other words, they could not walk independently. At this stage, many patients transfer to recuperation types of care facilities. We are planning to improve recruitment methods so that early cases can be registered. Patient tracking at advanced stages could also be a future function of such a registry. 
A. $n=16$

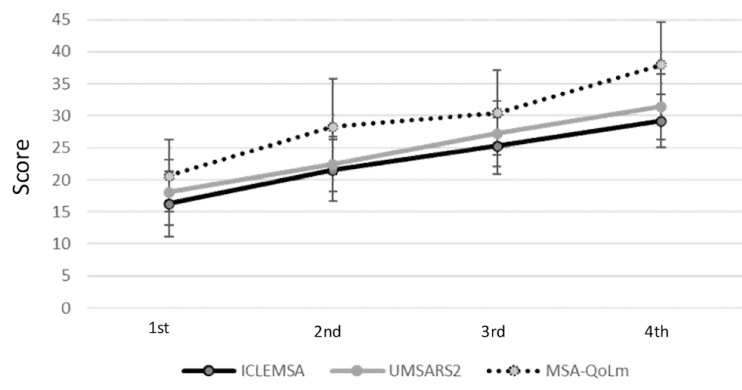

B. UMSARS part II

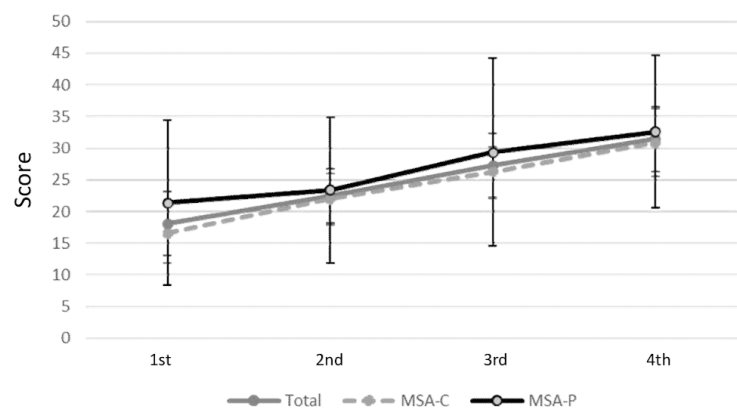

C. MSA-QoL motor score

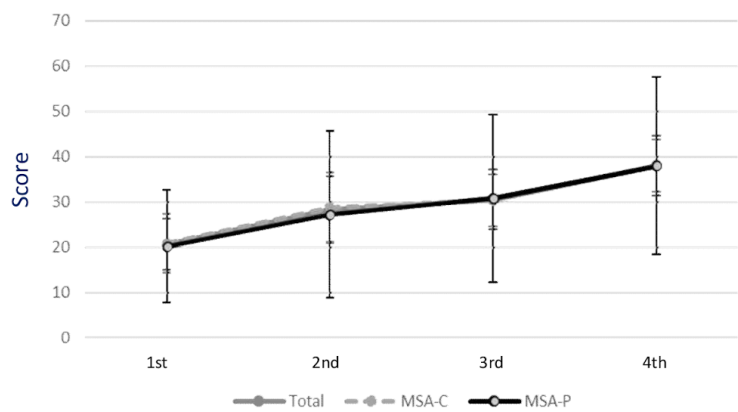

D. ICLEMSA

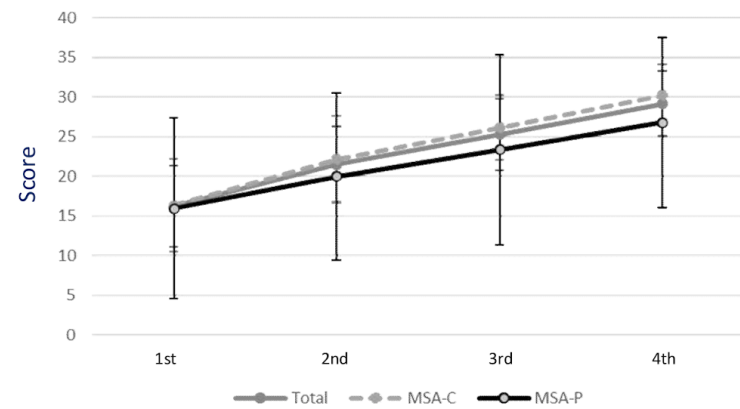

E. Standardized means of scale scores

\begin{tabular}{lcccc}
\hline Items & Baseline, 1 y later & $1-2 y$ later & $2-3$ y later & $3-4$ y later \\
\hline UMSARS part II $(n)$ & $0.951(67)$ & $0.968(34)$ & $0.587(15)$ & $2.828(3)$ \\
\hline ICLEMSA $(n)$ & $0.868(69)$ & $0.876(36)$ & $0.951(16)$ & $0.889(3)$ \\
\hline MSA-QoL $(n)$ & $0.858(66)$ & $0.589(30)$ & $0.752(14)$ & $7.778(2)$ \\
\hline BMI $(n)$ & $-0.281(62)$ & $-0.306(30)$ & $-0.244(13)$ & $0.826(2)$ \\
\hline
\end{tabular}

F. UMSARS-part II score changes by drug use

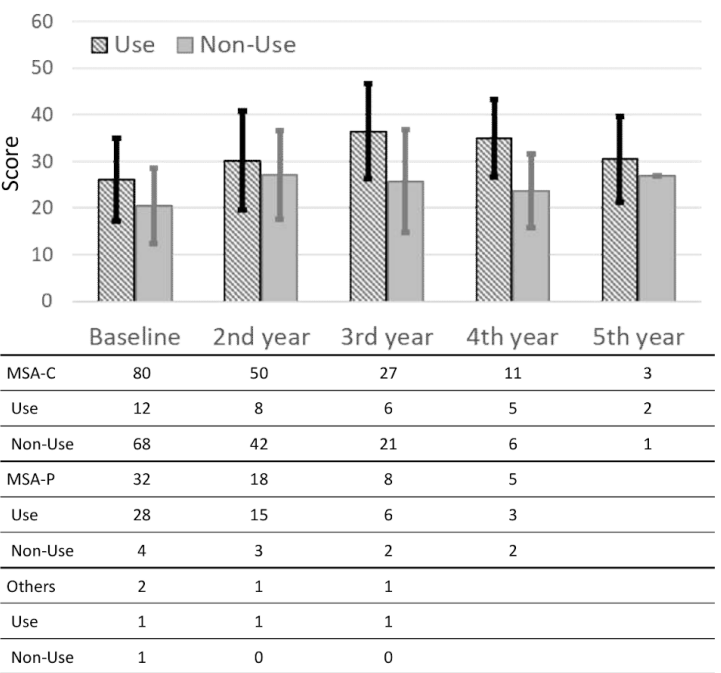

Figure 3 Changes in various scales over 4-5 years and standardised response mean of scale scores. (A) Changes in 16 cases that could be followed continuously up to the fourth evaluation. Black solid line: ICLEMSA; grey line: UMSARS Part II (UMSARS2); dotted line: MSA-QoL motor score (MSA-QoLm). (B-D) Score changes in UMSARS Part II (B), MSA-QoL motor score (C) and ICLEMSA (D) in 16 cases, MSA-C and MSA-P. Grey solid line: all 16 cases; dotted line: MSA-C; black line: MSA-P. Each data marker suggests the average and 95\% Cl. (E) Standardised response mean of scale scores. (F) UMSARS Part II score changes by drug use. Each bar shows the average score of each evaluation and the error bar indicates the SD. Shaded bars indicate levodopa use, and grey bars indicate levodopa non-use. The table shows the number of patients using levodopa who could be evaluated by the UMSARS. BMI, body mass index; ICLEMSA, Items That Change Largely in the EarlyStage Multiple System Atrophy; MSA, multiple system atrophy; MSA-C, cerebellar symptoms predominant type MSA; MSA-P, parkinsonism predominant type MSA; MSA-QoL, Multiple System Atrophy Health-Related Quality of Life scale; UMSARS, Unified Multiple System Atrophy Rating Scale.

MSA-P had higher UMSARS Part II scores overall than did MSA-C, and it was reconfirmed that MSA-P is the more severe condition. ${ }^{29}$ Higher scores on ICLEMSA for MSA-C than for MSA-P may indicate that this scale is relatively sensitive to ataxia. It has been pointed out in a previous report that in MSA, ataxia is more pronounced in the lower limbs, ${ }^{28}$ and it is thought that this feature is sensitively detected by ICLEMSA, which emphasises standing and walking. As the number of cases in our registry increases, more appropriate interpretations could come to light. The fact that the BMI changed little was described in a previous report. These results show that, unlike amyotrophic lateral sclerosis, no weight loss occurs during the advanced stages of MSA, but rather it might increase, even though calorie consumption decreases.

A possible reason for the higher UMSARS score in the levodopa group may be that levodopa was used in relatively severe cases. Most MSA-C cases tended to use taltirelin and not levodopa, and MSA-P cases tended to use levodopa and not taltirelin, resulting in drug use reflecting 
A

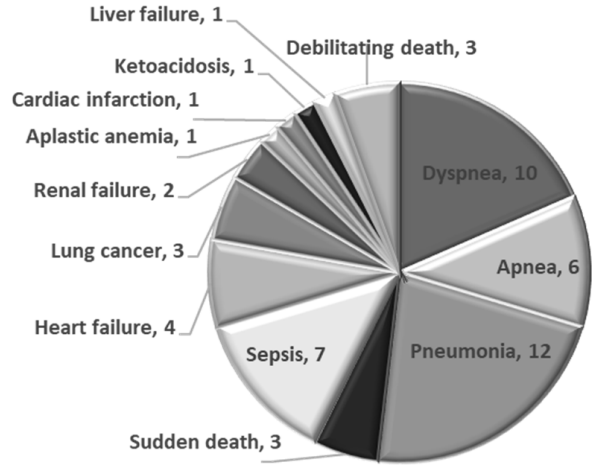

B

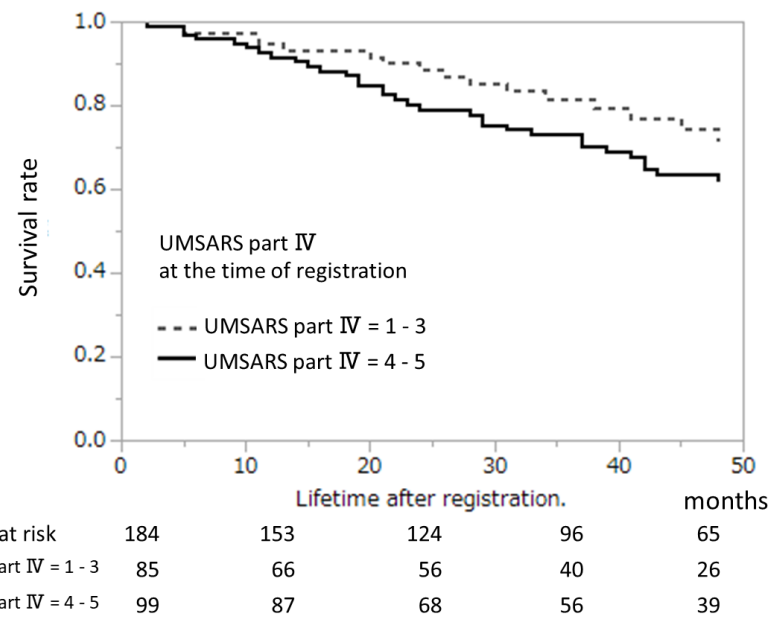

C

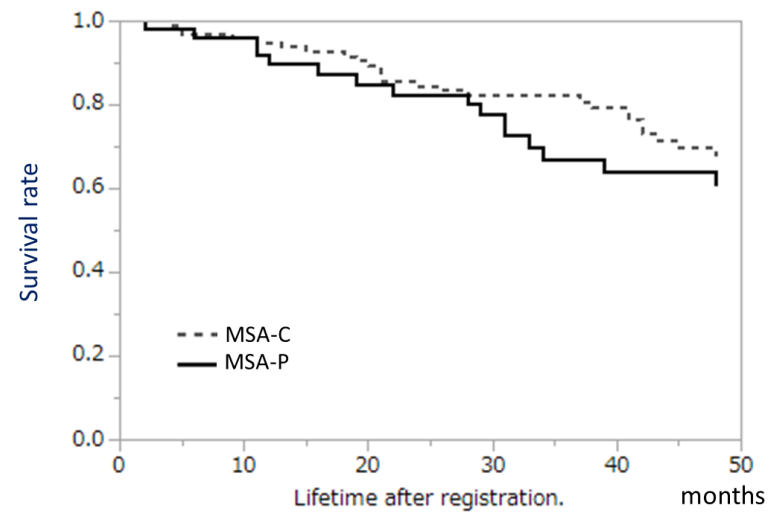

Number at risk MSA-C

MSA-P

162

132
90
42

107

72

35

Figure 4 Causes of death and Kaplan-Meier curves. (A) Causes of death (when known); (B) Kaplan-Meier curve by UMSARS Part IV at the time of registration; and (C) KaplanMeier curve by MSA-C and MSA-P at the time of registration. MSA, multiple system atrophy; MSA-C, cerebellar symptoms predominant type MSA; MSA-P, parkinsonism predominant type MSA; UMSARS, Unified Multiple System Atrophy Rating Scale.

MSA-C versus MSA-P status over time in this study population. In Japan, taltirelin and protirelin, the thyrotropinreleasing hormone analogues, were covered by insurance for spinocerebellar degeneration (SCD), which might also affect the fact that these drugs were used in many MSA-C cases. It is thought that the attending doctor will

not prescribe taltirelin if there are no ataxia symptoms and will not prescribe levodopa if there are no parkinsonian symptoms. In addition, the levodopa doses seen in this Asian study were smaller than those seen in Europe and the USA, which we also consider to have influenced the high MSA-P scores. The association between drug use, symptoms and severity needs to be further examined in the future.

More than half of the deaths we recorded were due to respiratory disorders. If measures were taken to prevent these in cases of MSA, the prognosis could be improved. $\mathrm{ADL}$ at registration and subsequent survival were not necessarily correlated in the Kaplan-Meier curves. Basically, it is assumed that the better the ADL, the longer the survival. However, the reason that the group with UMSARS Part IV score of 1 did not always have a favourable prognosis may be the small number of cases or the effect of sudden death. As a previous report has described, sudden death in MSA could occur at a relatively early stage. ${ }^{19}$

The limitations of this study are as follows. As is often the case with MSA, the diagnosis changes during the course of the disease in some patients. It could be difficult to track such diagnostic changes accurately, as diagnoses changed from SCD to MSA-C, and from Parkinson's disease and parkinsonism to MSA-P, and vice versa. In addition, patients with MSA are often transferred to longterm care facilities at the advanced stage. The number of registered cases may then be insufficient because it is difficult for neurologists to treat MSA right up to the end of life. In that case, non-neurologists often provide medical care and their evaluation may differ from that of the neurologist. Another limitation is that pathological findings were not obtained and investigation into the cause of death remains insufficient. It is desirable for the disease registry to be supplemented by systems for obtaining an accurate diagnosis by autopsy. It is possible that the neurologist cannot be fully involved in the terminal stages of the disease, which may bias the diagnostic MSA subtyping.

In conclusion, we report on the progress of Asia's first large-scale MSA prospective cohort study. This regional MSA registry can provide useful epidemiological information from continuing research activities. In Japanese patients with MSA, the proportion of MSA-C was initially larger, but symptoms of parkinsonism increased over time. A change of disease subtype from MSA-C to MSA-P occurred in 11 cases, but not vice versa. Parkinsonism suggested a poor prognosis. The possible benefits of incorporating pathological findings into the study and the association between drug use and severity need further investigation. The management of MSA requires caution due to the possibility of sudden death even in the early stages of the disease.

Author affiliations

${ }^{1}$ Department of Neurology, Hokkaido University Graduate School of Medicine,

Sapporo, Hokkaido, Japan 
${ }^{2}$ Department of Health Crisis Management, National Institute of Public Health, Wako, Saitama, Japan

${ }^{3}$ Department of Clinical Pharmacology, Tokai University School of Medicine, Isehara, Kanagawa, Japan

${ }^{4}$ Department of Biostatistics, Faculty of Medicine and Graduate School of Medicine, Hokkaido University, Sapporo, Hokkaido, Japan

${ }^{5}$ Department of Neurology, Date Red Cross Hospital, Date, Hokkaido, Japan

${ }^{6}$ Cardiovascular, Respiratory and Neurology Division, Department of Internal Medicine, Asahikawa Medical University, Asahikawa, Hokkaido, Japan

${ }^{7}$ Department of Medical Service, Aizen Hospital, Sapporo, Hokkaido, Japan

${ }^{8}$ Department of Neurology, Oji General Hospital, Tomakomai, Hokkaido, Japan

${ }^{9}$ Department of Neurology, Nakamura Memorial Hospital, Sapporo, Hokkaido, Japan

${ }^{10}$ Hokuyukai Neurological Hospital, Sapporo, Hokkaido, Japan

${ }^{11}$ Department of Public Health, Faculty of Medicine and Graduate School of

Medicine, Hokkaido University, Sapporo, Hokkaido, Japan

${ }^{12}$ Department of Neurology, Sapporo Medical University School of Medicine,

Sapporo, Hokkaido, Japan

${ }^{13}$ Hokkaido University Hospital Clinical Research and Medical Innovation Center,

Sapporo, Hokkaido, Japan

${ }^{14}$ Hokkaido Medical Center, Sapporo, Hokkaido, Japan

${ }^{15}$ Hakodate Central General Hospital, Hakodate, Hokkaido, Japan

Acknowledgements We would like to thank the Department of Health and Welfare, Hokkaido Government, Sapporo City Public Health Office, HoRC-MSA study group and the patients for their participation in this study. HoRC-MSA study group: Ikuko Iwata, Shinichi Shirai, Masahiro Wakita, Katsuki Eguchi (Hokkaido University Hospital), Shigehisa Ura, Hisashi Uwatoko, Kazuto Yoshida (Asahikawa Red Cross Hospital), Hideki Houzen, Takahiro Kano (Obihiro Kosei Hospital), Toshiyuki Fukazawa, Atsushi Kawashima (Sapporo Neurology Hospital), Hiroyuki Soma, Michio Nonaka, Fumio Moriwaka (Hokuyukai Neurological Hospital), Shin Hisahara, Shuichiro Suzuki, Akihiro Maatsumura, Daisuke Yamamoto, Jun Kawamata (Sapporo Medical University), Masahiro Mizobuchi (Nakamura Memorial Hospital), Nobuhito Murakami (Nakamura Memorial Minami Hospital), Takayuki Katayama (Asahikawa Municipal Hospital), Kikuchi Susumu, Ryutaro Ochi, Takayoshi Hozuki (Sapporo Shirakabadai Hospital), Naoya Minami (Hokkaido Medical Center), Takashi Kimura, Kenta Nomura (Asahikawa Medical Center), Yasunori Mito (Sapporo Municipal Hospital), Yasunori Maruo (Hakodate Municipal Hospital), Ryuji Miyagishi (Hokusei Memorial Hospital), Akihisa Matsumoto (Jozankei Hospital), Kazumasa Sudo (Sasson Hospital), Kazuo Yazaki (Seimeikan Clinic), Hiroyasu Furuyama (Sapporo Yamanoue Hospital), Kohei Echizenya (Echizenya Neurology Clinic), Tsukasa Saito (Asahikawa Medical University), Kazuki Hashimoto (Asahikawa Neurology Clinic), Satoshi Koyama (Asahikawa Rehabilitation Hospital), Tatsuro Sato (Hakodate Medical Association Hospital), Chikumi Otsuka (Muroran Neurology Clinic), Kyoko Hamada (Shinsapporo Neurosurgery Hospital), Yusuke Moriya (Aizen Hospital), Mayumi Kitagawa (Sapporo Teishinkai Hospital), Hajime Kamada (Hokuto Hospital), Souichi Hashimoto (Heiseikai Hospital), Kenshi Sasaki, Shinya Masui (Tokachi Neurosurgery Hospital), Yumiko Yoshikawa (Shinsapporo Paolo Hospital), Kimiko Takahashi (Sapporo Chuo Family Clinic), Mitsuhiro Tada (Rumoi City Hospital), Tetsuro Yanagihara (Ebetsu Shinkeinaika) and many other doctors in Hokkaido. We would like to thank Editage (www.editage.com) for English language editing.

Contributors Study concept and design: IY, KeS, HS. Acquisition, analysis or interpretation of data: MM, IY, KeS, HS. Data acquisition: MM, IY, TM, JS, HU, KaS, AsT, SS, SK. Management and analysis of data: MM, KeS, NN, AkT, NS. Drafting of the manuscript: MM, IY, KeS. Review of the manuscript for intellectual content: IY, KeS, YK, NN, TM, JS, HU, KaS, AsT, AKT, SS, NS, SK, HS.

Funding This research is supported by the Practical Research Project for Rare/Intractable Diseases from The Japan Agency for Medical Research and Development (AMED) and the Research Committee on the Medical Basis of Motor Ataxias, Health and Labour Sciences Research Grants, The Ministry of Health, Labour and Welfare, Japan.

Competing interests None declared.

Patient and public involvement Patients and/or the public were involved in the design, or conduct, or reporting, or dissemination plans of this research. Refer to the Materials and methods section for further details.

Patient consent for publication Not required.

Ethics approval Full written informed consent was obtained from all participants. This study was approved by the Institutional Review Board of Hokkaido University Hospital.
Provenance and peer review Not commissioned; externally peer reviewed.

Data availability statement Data are available upon reasonable request. If anyone applies for use of research data, the research group will consider permission for use.

Open access This is an open access article distributed in accordance with the Creative Commons Attribution Non Commercial (CC BY-NC 4.0) license, which permits others to distribute, remix, adapt, build upon this work non-commercially, and license their derivative works on different terms, provided the original work is properly cited, appropriate credit is given, any changes made indicated, and the use is non-commercial. See: http://creativecommons.org/licenses/by-nc/4.0/.

\section{ORCID iD}

Masaaki Matsushima http://orcid.org/0000-0001-6585-3686

\section{REFERENCES}

1 Graham JG, Oppenheimer DR. Orthostatic hypotension and nicotine sensitivity in a case of multiple system atrophy. J Neurol Neurosurg Psychiatry 1969;32:28-34.

2 Quinn N. Multiple system atrophy--the nature of the beast. J Neurol Neurosurg Psychiatry 1989::78-89.

3 Wakabayashi K, Yoshimoto M, Tsuji S, et al. Alpha-synuclein immunoreactivity in glial cytoplasmic inclusions in multiple system atrophy. Neurosci Lett 1998;249:180-2.

4 Orosz F, Kovács GG, Lehotzky A, et al. Tppp/P25: from unfolded protein to misfolding disease: prediction and experiments. Biol Cell 2004;96:701-11.

5 Kaufman E, Hall S, Surova Y, et al. Proinflammatory cytokines are elevated in serum of patients with multiple system atrophy. PLoS One 2013;8:e62354.

6 Mitsui J, Matsukawa T, Ishiura H. Mutations in COQ2 in familial and sporadic multiple-system atrophy the multiple-system atrophy research collaboration. N Engl J Med 2013;369:233-44.

7 Holmberg B, Johansson J-O, Poewe W, et al. Safety and tolerability of growth hormone therapy in multiple system atrophy: a doubleblind, placebo-controlled study. Mov Disord 2007;22:1138-44.

8 Bensimon G, Ludolph A, Agid Y, et al. Riluzole treatment, survival and diagnostic criteria in Parkinson plus disorders: the NNIPPS study. Brain 2009;132:156-71.

9 Low PA, Robertson D, Gilman S, et al. Efficacy and safety of rifampicin for multiple system atrophy: a randomised, double-blind, placebo-controlled trial. Lancet Neurol 2014;13:268-75.

10 Dodel R, Spottke A, Gerhard A, et al. Minocycline 1-year therapy in multiple-system-atrophy: effect on clinical symptoms and [(11)C] (R)PK11195 PET (MEMSA-trial). Mov Disord 2010;25:97-107.

11 Poewe W, Seppi K, Fitzer-Attas CJ, et al. Efficacy of rasagiline in patients with the parkinsonian variant of multiple system atrophy: a randomised, placebo-controlled trial. Lancet Neurol 2015;14:145-52.

12 Levin J, Maaß S, Schuberth M, et al. Safety and efficacy of epigallocatechin gallate in multiple system atrophy (PROMESA): a randomised, double-blind, placebo-controlled trial. Lancet Neurol 2019;18:724-35.

13 Lee $\mathrm{PH}$, Lee JE, Kim H-S, et al. A randomized trial of mesenchymal stem cells in multiple system atrophy. Ann Neurol 2012;72:32-40.

14 Luo Y, Fan Y, Zhou B, et al. Generation of induced pluripotent stem cells from skin fibroblasts of a patient with olivopontocerebellar atrophy. Tohoku J Exp Med 2012;226:151-9.

15 Mitsui J, Koguchi K, Momose T, et al. Three-year follow-up of highdose ubiquinol supplementation in a case of familial multiple system atrophy with compound heterozygous COQ2 mutations. Cerebellum 2017;16:664-72.

16 May S, Gilman S, Sowell BB, et al. Potential outcome measures and trial design issues for multiple system atrophy. Mov Disord 2007:22:2371-7.

17 Wenning GK, Geser F, Krismer F, et al. The natural history of multiple system atrophy: a prospective European cohort study. Lancet Neurol 2013:12:264-74.

18 Low PA, Reich SG, Jankovic J, et al. Natural history of multiple system atrophy in the USA: a prospective cohort study. Lancet Neurol 2015;14:710-9.

19 Yabe I, Soma H, Takei A, et al. MSA-C is the predominant clinical phenotype of MSA in Japan: analysis of 142 patients with probable MSA. J Neurol Sci 2006;249:115-21.

20 Watanabe H, Saito Y, Terao S, et al. Progression and prognosis in multiple system atrophy: an analysis of 230 Japanese patients. Brain 2002;125:1070-83. 
21 Zhang L, Cao B, Zou Y, et al. Causes of death in Chinese patients with multiple system atrophy. Aging Dis 2018;9:102-8.

22 Sakushima K, Nishimoto N, Nojima M, et al. Epidemiology of multiple system atrophy in Hokkaido, the northernmost island of Japan. Cerebellum 2015;14:682-7.

23 Wenning GK, Tison F, Seppi K, et al. Development and validation of the unified multiple system atrophy rating scale (UMSARS). Mov Disord 2004;19:1391-402.

24 Gilman S, Wenning GK, Low PA, et al. Second consensus statement on the diagnosis of multiple system atrophy. Neurology 2008;71:670-6.

25 Schrag A, Selai C, Mathias C, et al. Measuring health-related quality of life in MSA: the MSA-QoL. Mov Disord 2007:22:2332-8.
26 Matsushima M, Yabe I, Takahashi I, et al. Validity and reliability of a pilot scale for assessment of multiple system atrophy symptoms. Cerebellum Ataxias 2017:4:11.

27 Cohen J. Statistical power analysis for the behavioral sciences. 2nd edn. Hillsdale: NJ Lawrence Erlbaum Associates, Publishers, 1988.

28 Hirayama K, Kita K. [Clinical features of "multiple system atrophies"-olivo-ponto-cerebellar atrophy, striato-nigral degeneration and Shy-Drager syndrome]. Rinsho Shinkeigaku 1985;25:1196-203.

29 Matsushima M, Yabe I, Oba K, et al. Comparison of different symptom assessment scales for multiple system atrophy. Cerebellum 2016:15:190-200. 